\title{
Association between JAK1 gene polymorphisms and susceptibility to allergic rhinitis
}

\author{
Yang Shen, ${ }^{1 *}$ Yun Liu,,${ }^{1,2^{*}}$ Xia Ke,${ }^{1}$ Hou-Yong Kang, ${ }^{1}$ Guo-Hua Hu${ }^{1}$ and Su-Ling Hong ${ }^{1}$
}

\section{Summary}

Objectives: Allergic rhinitis (AR) is an inflammatory disorder of the upper airway. Janus kinase 1 (JAK1), a member of JAK family, has recently been found to participate in the immune response and the development of allergic airway disease. This study was performed to evaluate the potential association of JAK1 polymorphisms with $A R$ in a Chinese Han population.

Methods: A case-control study was performed in 450 Chinese AR patients and 615 healthy controls. Three SNPs in the JAK1 gene, rs3790532, rs310241 and rs2780815, were analyzed using a polymerase chain reactionrestriction fragment length polymorphism assay (PCR-RFLP).

Results: An association was detected between SNP rs310241 in the JAK1 gene and AR in a Chinese Han population. However, no significant association was observed between the polymorphisms rs3790532 and rs2780815 and AR. For rs310241, the CC genotype and the $C$ allele significantly increased the risk of $A R$. Furthermore, we found that the ACG haplotype in JAK1 gene was positively correlated with AR, while the GTG haplotype was associated with a significantly decreased risk of AR.

Conclusion: This study indicates that the JAK1 rs310241 C-related genotype and allele are involved in AR susceptibility, making them potentially useful genetic biomarkers for AR susceptibility in the Chinese Han population. (Asian Pac J Allergy Immunol 2016;34:124-9)

\footnotetext{
From 1. Department of Otorhinolaryngology, The First

Affiliated Hospital of Chongqing Medical University,

Chongqing, People's Republic of China

2. Chongqing Key Laboratory of Ophthalmology, Chongqing,

People's Republic of China

Corresponding author: Su-Ling Hong

E-mail: sy_smile@sina.cn

Submitted date: 10/2/2015

Accepted date: 23/9/2015
}

Keywords: Janus kinase 1, polymorphism, allergy, rhinitis, susceptibility

\section{Introduction}

Allergic rhinitis (AR) is an IgE-mediated inflammation of the upper airway, which is induced by allergens and regulated by $\mathrm{T}$ cells. AR has an estimated worldwide incidence of $10-20 \% .^{1}$ Our previous epidemiological investigations showed that in western China, the prevalence of self-reported AR was $32.30 \%$ (Chongqing), $34.3 \%$ (Chengdu), 37.9\% (Urumqi), and $30.3 \%$ (Nanning). ${ }^{2} \mathrm{AR}$ has a major impact on quality of life by causing unpleasant symptoms such as sneezing, nasal congestion, nasal pruritus, rhinorrhea and obstruction of the nasal passages. Furthermore, AR is a known risk factor for comorbid conditions such as asthma, rhinosinusitis, nasal polyposis, and sleep disorders, resulting in important medical and social problems. ${ }^{3}$, 4

Over the last two decades, the pathogenesis of AR has been widely studied and genetic factors are thought to be major players affecting the development, severity and treatment of AR. ${ }^{5,} 6$ Genetic studies showed that single nucleotide polymorphisms (SNPs) contribute greatly to susceptibility to AR. ${ }^{7,} 8$ Our recent study also demonstrated important associations between polymorphisms in the IL-23R and IL-27 genes and AR in the Han Chinese population. ${ }^{9}{ }^{10}$ However, the details of the underlying pathogenic mechanisms remain unclear.

Janus kinase 1 (JAK1), first identified in 1991, is a member of a new class of protein-tyrosine kinases. ${ }^{11}$ It is a key member in both the $\alpha / \beta$ interferon (IFN $\alpha / \beta)$ and IFN $\gamma$ signaling pathways. ${ }^{12}$ A recent study found that the JAK $1 / 3$ signaling pathways are key initiators of Th2 differentiation and lung allergic responses. ${ }^{13}$ Furthermore, Chen et al. found that some medication might be used to improve airway allergy and asthma through regulation of the JAK1-STAT6 signaling pathway. ${ }^{14}$ Thus, we suspect that some JAK1 genetic variations might play roles in the complex pathogenesis of AR, which is an important allergic disease in the upper 
airway. The JAK1 gene, with 25 exons and encoding a protein of 1154 amino acids, is located on chromosome 1p31.3. It has been found that JAK1 mutations or SNPs might be functional and contribute to asthma, Behçet's disease, Dengue hemorrhagic fever, Hepatitis B virus infection and human hepatocellular carcinoma. ${ }^{15-19}$

However, to date, no studies have examined the role of JAK1 SNPs in the development of AR. Thus, in this study, we attempted to gain a better understanding of the genetic influence of JAK1 on AR. We have identified three possible variation sites of JAK1, including rs3790532, rs310241 and rs2780815. To determine whether these JAK1 SNPs are associated with susceptibility to AR, we analyzed their frequencies in genomic DNA isolated from AR patients and healthy controls.

\section{Methods}

\section{Patients and Healthy Controls}

In total, 450 patients (231 men, 219 women) between 4 and 70 years of age were recruited from October 2012 to August 2013. All patients were identified by and treated at the outpatient clinic of the Department of Otolaryngology Head and Neck Surgery at the First Affiliated Hospital of Chongqing Medical University, Chongqing, China. The diagnosis of AR was based on the patients' medical history and symptoms and the presence of a positive skin prick test (SPT, Allergopharma, Hamburg, Germany) in response to a panel of common allergens defined by the ARIA 2008 guidelines. ${ }^{20}$ The SPT results were diagnosed in accordance with the recommendations of the Subcommittee on Allergen Standardization and Skin Tests of the European Academy of Allergy and Clinical Immunology. ${ }^{21}$ A positive SPT result was defined as the formation of a wheal larger than or equal to one half the diameter of the histamine control wheal, and at least $3 \mathrm{~mm}$ larger than the diameter of the negative control wheal. A total of 18 inhaled allergens were tested, including house dust, grass, tree, mold, food, and cat and dog dander.

In contrast, patients with accompanying systemic disease were excluded from the study. For this, 615 healthy volunteers of the same ethnicity as the patients were recruited as the control group. The selection criteria for healthy volunteers were no chronic pathology, in particular, no history of allergy or respiratory pathology, no other systemic diseases, and no evidence of any family history of allergy. Furthermore, healthy volunteers matched cases for both age and gender.

\section{Ethics Statement}

The local ethics authorities (the Ethics Committee of the First Affiliated Hospital of Chongqing Medical University, Chongqing, China) provided permission and helped to obtain informed consent from all participants. Written informed consent was obtained from all participants. Informed consent was obtained from the next of kin, caretakers, or guardians of any minors participating in the study.

\section{DNA extraction}

Genomic DNA was extracted from EDTAanticoagulated peripheral blood leukocytes using the Wizard $\AA$ Genomic DNA Purification Kit method. Briefly, $300 \mu 1$ of blood was mixed with cell lysis solution. Leucocytes were spun down and lysed with Nuclei Lysis Solution. The pellet was separated out by the Protein Precipitation Solution and precipitated proteins were removed by centrifugation. Then, double-stranded DNA was separated out by methyl alcohol. The DNA on the EP tube was dissolved in $100 \mu$ DNA Rehydration Solution.

\section{SNP Selection and Genotyping}

We selected the rs3790532, rs310241 and rs2780815 SNPs in the JAK1 gene as candidate sites which have been demonstrated to be associated with certain immune-related diseases. ${ }^{16}$ SNPs rs 3790532 , rs310241 and rs2780815 are located within introns. These three SNPs were genotyped using the polymerase chain reaction-restriction fragment length polymorphism (PCR-RFLP) method. The PCR products were incubated with restriction enzymes for 1 or $1-16 \mathrm{~h}$. The obtained digestion products were visualized on a $4 \%$ agarose gel and stained with Gold View (SBS Genentech, Beijing, China). To confirm the genotyping results, PCRamplified DNA samples were examined by direct sequencing ( $20 \%$ of all the blood samples), and the results were $100 \%$ concordant.

\section{Statistical analysis}

All statistical analyses were performed using SPSS version 18.0 software (SPSS Inc., Chicago, Illinois, USA). $\mathrm{P}$ values less than 0.05 were considered statistically significant. To evaluate the quality of the genotyping data, the Hardy-Weinberg equilibrium (HWE) for SNP genotype frequencies was tested using a chi-square teat $\left(\chi^{2}\right.$ test). Allele and genotype frequencies between patients with $\mathrm{AR}$ and the controls were compared by $\chi^{2}$ test. The P- 
values were corrected $\left(\mathrm{PC}^{*}\right)$ with the Bonferroni correction by multiplying by the number of analyses performed. The online software platform SHEsis (http://analysis2.bio-x.cn/myanalysis.php) was used to analyze the haplotype and probabilities. The association between genotypes/alleles and AR risk was estimated by calculating odds ratios (OR) and $95 \%$ confidence intervals (CI).

\section{Results}

\section{Clinical characteristics of the study participants}

The demographics of the cases and controls enrolled in this study are shown in Table 1 . There were no significant differences between the cases and controls in terms of the mean age and gender distribution. $275(61.2 \%)$ patients were sensitive to house dust mites, $83(18.4 \%)$ were sensitive to tree pollen and $92(20.4 \%)$ were sensitive to multiple allergens.

\section{Genotype distribution of the JAK1 polymorphisms}

Our results showed that the JAK1 SNPs rs3790532, rs310241 and rs2780815 were in HardyWeinberg equilibrium in the cases and controls $(\mathrm{P}>0.01)$. The genotype and allele frequencies of the three tested JAK1 polymorphisms are shown in Table 2. The call rate for the three examined SNPs was $100 \%$.

The results showed that there were obvious differences between the AR patients and controls concerning the rs 310241 frequencies. A significantly increased prevalence of the rs310241 CC genotype and $\mathrm{C}$ allele were found in $\mathrm{AR}$ patients compared to controls $(\mathrm{P}=0.00003$, OR 2.38, 95\%CI 1.64-3.47; $\mathrm{P}=0.036$, OR $1.22,95 \%$ CI 1.03-1.45, respectively). However, no significant differences were found in the genotype frequency of the rs3790532 and rs2780815 SNPs between the AR cohort and the control group.

\section{Haplotype analysis of the JAK1 gene}

Haplotype analyses were performed using the Haploview v3.32 program and the online software platform SHEsis. The six possible haplotype frequencies are shown in Table 3. The major Grs3790532Trs310241Grs2780815 haplotype, constructed by SNPs including rs3790532, rs310241 and rs 2780815 , accounted for $61.8 \%$ and $67.1 \%$ of these six haplotypes in the cases and the controls, respectively. We found that the distribution of the Ars3790532Crs310241Grs2780815 haplotypes was notably increased in the AR patients compared to the controls and that the distribution of the
Table 1. Selected clinical features and demographic characteristics of study population and controls.

\begin{tabular}{lll}
\hline Characteristics & $\boldsymbol{A R}(\mathbf{N}=\mathbf{4 5 0})$ & Control(N=615) \\
\hline Gender[male/female] & $231 / 219$ & $298 / 317$ \\
Age[mean(range)]years & $28.74(4-70)$ & $34.23(7-75)$ \\
Allergen & & \\
$\quad$ House dust mite & $275(61.2 \%)$ & - \\
Pollen & $83(18.4 \%)$ & - \\
$\quad$ Multiple allergens & $92(20.4 \%)$ & - \\
\hline
\end{tabular}

Grs3790532Trs310241Grs2780815 haplotypes was significantly reduced. The Ars3790532Crs310241 Grs2780815 haplotype in the JAK1 gene was positively correlated with AR, while the Grs3790532Trs310241Grs2780815 haplotype was associated with a significantly decreased risk of AR.

\section{Discussion}

In this study, we investigated the JAK1 gene polymorphisms in AR and demonstrated a novel association between the SNP rs310241 in the JAK1 gene and $\mathrm{AR}$ in a Chinese Han population. For rs310241, the CC genotype and the $\mathrm{C}$ allele significantly increased the risk of AR. Furthermore, we found that the ACG haplotype in the JAK1 gene was positively correlated with AR, while the GTG haplotype was negatively correlated with AR. To the best of our knowledge, this is the first study to evaluate an association between the JAK1 SNP rs310241 and AR in a Chinese Han population.

$\mathrm{AR}$, a common inflammatory disorder of the upper airway, is the result of a complex interplay between multiple genetic and environmental factors resulting in dysregulation of the immune system. ${ }^{22}$ To explore the genetic influences of JAK1 on AR, we chose rs3790532, rs310241 and rs2780815 in the JAK1 gene as candidate sites, based on the results of previous studies. For example, a recent study showed that three JAK1 gene SNPs including rs2780815, rs310241 and rs3790532 were strongly associated with Behçet's disease in Chinese Han. ${ }^{16}$ Meanwhile, $\mathrm{Hu}$ et al. reported that three SNPs, rs310230, rs310236, and rs310241, in JAK1 were significantly associated with VKH syndrome. ${ }^{23}$ In this study, our data demonstrated an association between the SNP rs310241 in the JAK1 gene and AR. However, no significant associations were observed between rs3790532 and rs2780815 and AR. These results are partially consistent with those reported in the above studies and suggest that the 
Table 2. The genotype and allele frequencies of JAK1 polymorphisms rs3790532, rs310241, rs 2780815 in AR patients and controls.

\begin{tabular}{lllllll}
\hline SNP & $\begin{array}{l}\text { Genotype } \\
\text { Allele }\end{array}$ & $\begin{array}{l}\text { AR }(\%) \\
(\boldsymbol{n}=\mathbf{4 5 0})\end{array}$ & $\begin{array}{l}\text { Controls }(\%) \\
(\boldsymbol{n}=\mathbf{6 1 5})\end{array}$ & $\boldsymbol{P}$ Value & Pc* Value & Adjusted OR (95\% CI) \\
\hline rs3790532 & AA & $70(15.6)$ & $68(11.1)$ & 0.031 & NS & $1.407(1.031-1.920)$ \\
& AG & $204(45.3)$ & $262(42.6)$ & NS & NS & $1.064(0.928-1.220)$ \\
& GG & $176(39.1)$ & $285(46.3)$ & 0.019 & NS & $0.844(0.731-0.974)$ \\
& A & $172(38.0)$ & $199(32.4)$ & 0.047 & NS & $1.181(1.003-1.392)$ \\
rs310241 & G & $278(62.0)$ & $416(67.6)$ & & & \\
& CC & $68(15.2)$ & $39(6.4)$ & $\mathbf{0 . 0 0 0 1}$ & $\mathbf{0 . 0 0 0 3}$ & $2.383(1.639-3.465)$ \\
& CT & $206(45.7)$ & $298(48.5)$ & NS & NS & $1.024(0.903-1.163)$ \\
& TT & $176(39.1)$ & $278(45.2)$ & $\mathbf{0 . 0 4 7}$ & NS & $0.865(0.749-1.000)$ \\
& C & $157(38.0)$ & $191(31.0)$ & $\mathbf{0 . 0 2 4}$ & NS & $1.218(1.027-1.44527$ \\
rs2780815 & T & $258(62.0)$ & $424(79.0)$ & & & \\
& TT & $27(6.1)$ & $42(6.9)$ & NS & NS & $0.953(0.597-1.520)$ \\
& GT & $135(30.1)$ & $177(28.7)$ & NS & NS & $1.042(0.864-1.258)$ \\
& GG & $288(63.8)$ & $396(65.4)$ & NS & NS & $0.994(0.908-1.088)$ \\
& T & $96(21.3)$ & $130(21.2)$ & NS & NS & $1.009(0.799-1.275)$ \\
\hline
\end{tabular}

rs310241 polymorphism in the JAK1 gene may be associated with AR susceptibility.

JAK1 has a wide variety of immunosuppressive activities. It is the gatekeeper in the JAK-STAT pathway, which is a major suppressor of some cytokines during cell-signaling process. For the JAK1 gene, recent studies found that JAK1 genetic variations are associated with the risk of Crohn's disease and leukemia, ${ }^{24,25}$ and that JAK1 expression levels might be regulated by DNA methylation. ${ }^{26}$

In this study, our haplotype analysis indicated that the $\mathrm{A}_{\mathrm{r} 33790532} \mathrm{C}_{\mathrm{r} 3310241} \mathrm{G}_{\mathrm{rr} 2780815}$ haplotype in the JAK1 gene was positively correlated with AR, but that the $\mathrm{G}_{\mathrm{rs} 3790532} \mathrm{~T}_{\mathrm{rs} 310241} \mathrm{G}_{\mathrm{rs} 2780815}$ haplotype was negatively correlated with AR. It is therefore likely that people with the $\mathrm{A}_{\mathrm{rs} 3790532} \mathrm{C}$ rs310241 $\mathrm{G}_{\mathrm{rs} 2780815}$ haplotype are at a higher risk of developing AR than people with other haplotypes. Conversely, people with the $\mathrm{G}_{\mathrm{rs} 3790532} \mathrm{~T}_{\mathrm{rs} 310241} \mathrm{G}_{\mathrm{rs} 2780815}$ haplotype would be more resistant to $A R$, suggesting that the $\mathrm{G}_{\mathrm{r} 33790532} \mathrm{~T}_{\mathrm{r} s 310241} \mathrm{G}_{\mathrm{r} s 2780815}$ haplotype in the JAK1 gene may play a protective role in the development of AR.

As for rs310241, which lies within an intronic region of the JAK1 gene, we believe that it is directly involved in AR susceptibility. Our data show that the CC genotype of rs310241 increases the risk of AR 2.38-fold, while the $\mathrm{C}$ allele of rs 310241 is associated with a 1.22 -fold increase in the risk of $A R$. This suggests that the $C$ allele may lead to AR susceptibility, and that people with the C allele in the rs310241 SNP of the JAK1 gene are more likely to develop AR. Furthermore, we found that the ACG haplotype in rs2780815, rs310241 and rs3790532 of JAK1 is associated with a 1.962-fold increase in the risk of AR. However, the GTG haplotype decreased the risk of AR 0.727-fold. We speculate that these changes in genotype and haplotype may affect gene splicing, transcription factor binding, or the non-coding RNA sequence, and might thereby influence the expression and function of certain proteins.

In the present study, we made many efforts to decrease the influence of confounding factors on the results. We selected AR patients and controls using strict guidelines and confirmed the genotyping results by direct sequencing. We observed a novel association between the rs310241 SNP of JAK1 and AR in a Chinese Han population. However, it is worth mentioning that there are some limitations to our study. First, we did not assess the level of JAK1 protein in the peripheral blood or perform a functional analysis study, so we cannot draw certain conclusions about the influence of these polymorphisms on the cytokine levels. Second, as it is well known that environmental factors are critical for the development of AR, further studies will be needed to clarify the genetic influence of JAK1 in 
Table 3. Haplotype frequencies of rs3790532, rs310241 and rs2780815 in the JAK1 gene.

\begin{tabular}{lccccc}
\hline \multicolumn{1}{c}{ Haplotype } & AR (\%) & Control(\%) & $\chi^{2}$ & P & Odds Ratio (95\%CI) \\
\hline ACG & $153.16(0.170)$ & $113.22(0.092)$ & 26.42 & $2.80 \times 10^{-7}$ & $1.962[1.512 \sim 2.545]$ \\
ACT & $188.84(0.210)$ & $255.55(0.208)$ & 37.65 & 0.83 & $0.977[0.791 \sim 1.208]$ \\
ATG & $1.84(0.002)$ & $23.78(0.019)$ & - & - & - \\
A T T & $0.16(0.000)$ & $5.45(0.004)$ & - & - & - \\
GCG & $0.00(0.000)$ & $7.23(0.006)$ & - & - & - \\
GTG & $556.00(0.618)$ & $824.77(0.671)$ & 11.80 & $0.60 \times 10^{-3}$ & $0.727[0.606 \sim 0.872]$ \\
\hline
\end{tabular}

AR pathogenesis, including gene-gene and geneenvironment interactions.

In summary, we have demonstrated an association between JAK1 gene polymorphisms and susceptibility to AR. Our results suggest that rs310241, but not rs2780815 and rs3790532, is a susceptibility allele for the development of AR in the Chinese Han population. However, more intensive studies are required to further clarify the molecular mechanisms and the complex interaction between environmental factors and JAK1 gene polymorphisms in AR. Generally, we hope that our study can provide a deeper insight into AR pathogenesis.

\section{Acknowledgements}

This study was supported by the National Natural Science Foundation of China (81470676) and National Key Clinical Specialties Construction Program of China.

\section{Conflicts of interest}

The authors declare no financial or other conflicts of interest regarding the content of this article.

\section{References}

1. Togias A. Rhinitis and asthma: evidence for respiratory system integration. J Allergy Clin Immunol. 2003;111:1171-83; quiz 1184.

2. Shen J, Ke X, Hong S, Zeng Q, Liang C, Li T, et al. Epidemiological features of allergic rhinitis in four major cities in Western China. J Huazhong Univ Sci Technolog Med Sci. 2011;31:433-40.

3. Young MC. Rhinitis, sinusitis, and polyposis. Allergy Asthma Proc. 1998;19:211-8.

4. Leynaert B, Neukirch F, Demoly P, Bousquet J. Epidemiologic evidence for asthma and rhinitis comorbidity. J Allergy Clin Immunol. 2000;106:S201-5.
5. Vercelli D. Genetic polymorphism in allergy and asthma. Curr Opin Immunol. 2003;15:609-13.

6. Joki-Erkkila VP, Karjalainen J, Hulkkonen J, Pessi T, Nieminen MM, Aromaa A, et al. Allergic rhinitis and polymorphisms of the interleukin 1 gene complex. Ann Allergy Asthma Immunol. 2003;91:275-9.

7. Tang XF, Tang HY, Sun LD, Xiao FL, Zhang Z, Li Y, et al. Genetic variant rs4982958 at 14q11.2 is associated with allergic rhi nitis in a Chinese Han population. J Investig Allergol Clin Immunol. 2012;22:55-62.

8. Qian F, Zhang Q, Zhou L, Ma G, Jin G, Huang Q, et al. Association between polymorphisms in IL17F and male asthma in a Chinese population. J Investig Allergol Clin Immunol. 2012;22:257-63.

9. Hu D, Hu G, Zhu J, Shen Y, Kang H, Hong S. Association between polymorphisms of the IL-23R gene and allergic rhinitis in a Chinese Han population. PLoS One. 2013;8:e63858.

10. Shen Y, Yuan XD, Hu D, Ke X, Wang XQ, Hu GH, et al. Association between interleukin-27 gene polymorphisms and susceptibility to allergic rhinitis. Hum Immunol. 2014;75(9):991995.

11. Wilks AF, Harpur AG, Kurban RR, Ralph SJ, Zürcher G, Ziemiecki A. Two novel protein-tyrosine kinases, each with a second phosphortransferase-related catalytic domain, define a new class of protein kinase. Mol Cell Biol. 1991;11(4):2057-65.

12. Kalvakolanu DV. Alternate interferon signaling pathways. Pharmacol Ther. 2003;100:1-29.

13. Ashino S, Takeda K, Li H, Taylor V, Joetham A, Pine PR, et al. Janus kinase $1 / 3$ signaling pathways are key initiators of $\mathrm{TH} 2$ differentiation and lung allergic responses. J Allergy Clin Immunol. 2014;133:1162-74.

14. Chen XH, Zhong NS, Zhang WD, Cao ZZ, He MZ, Luo Q,et al. Budesonide attenuates airway remodeling and modules the expression of Janus protein tyrosine kinase 1, and signal transducers and activators of transcription 6 in asthma: an experiment with mice. Zhonghua Yi Xue Za Zhi. 2007;87:1627-32.

15. Hsieh YY, Chang CC, Hsu CM, Wan L, Chen SY, Lin WH, et al. JAK-1 rs2780895 C-related genotype and allele but not JAK-1 
rs10789166, rs4916008, rs2780885, rs17127114, andrs3806277 are associated with higher susceptibility to asthma. Genet Test Mo Biomarkers. 2011;15:841-7.

16. Hou S, Qi J, Zhang Q, Liao D, Li Q, Hu K, et al. Genetic variants in the JAK1 gene confer higher risk of Behcet's disease with ocular involvement in Han Chinese. Hum genet. 2013;132:1049-58.

17. Silva LK, Blanton RE, Parrado AR, Melo PS, Morato VG, Reis EA et al. Dengue hemorrhagic fever is associated with polymorphisms in JAK1. Eur J Hum Genet. 2010;18:1221-7.

18. Chen K, Min H, Wu X, Zhu X, Li Z, Li H, et al. JAK1 gene polymorphisms are associated with the outcomes of hepatitis $\mathrm{B}$ virus infection, but not with alpha interferon therapy response in a Han Chinese population. Genet Test Mol Biomarkers. 2012;16:1206-10.

19. Xie HJ, Bae HJ, Noh JH, Eun JW, Kim JK, Jung KH, et al. Mutational analysis of JAK1 gene in human hepatocellular carcinoma. Neoplasma. 2009;56:136-40.

20. Bousquet J, Khaltaev N, Cruz AA, Denburg J, Fokkens WJ, Togias A, et al. Allergic Rhinitis and its Impact on Asthma(ARIA) 2008 update (in collaboration with the World Health Organization, GA(2)LEN and AllerGen). Allergy. 2008;63:8-160.
21. Position paper: Allergen standardization and skin tests. The European Academy of Allergology and Clinical Immunology. Allergy. 1993;48:48-82.

22. Dávila I, Mullol J, Ferrer M, Bartra J, del Cuvillo A, Montoro J, et al. Genetic aspects of allergic rhinitis. J Investig Allergol Clin Immunol. 2009; 19:25-31.

23. Hu K, Hou S, Li F, Xiang Q, Kijlstra A, Yang P. JAK1, but not JAK2 and STAT3, confers susceptibility to Vogt-Koyanagi-Harada (VKH) syndrome in a Han Chinese population. Invest Ophthalmol Vis Sci. 2013;54:3360-5.

24. Ferguson LR, Han DY, Fraser AG, Huebner C, Lam WJ, Morgan $\mathrm{AR}$, et al. Genetic factors in chronic inflammation: single nucleotide polymorphisms in the STAT-JAK pathway, susceptibility to DNA damage and Crohn's disease in a New Zealand population. Mutat Res. 2010;690:108-15.

25. Zhong Y, Chen B, Feng J, Cheng L, Li Y, Qian J, et al. The associations of Janus kinase-2 (JAK2) A830G polymorphism and the treatment outcomes in patients with acute myeloid leukemia. Leuk Lymphoma. 2010;51:1115-20.

26. Buslei R, Kreutzer J, Hofmann B, Schmidt V, Siebzehnrübl F, et al. Abundant hypermethylation of SOCS-1 in clinically silent pituitary adenomas. Acta Neuropathol. 2006;111:264-71. 